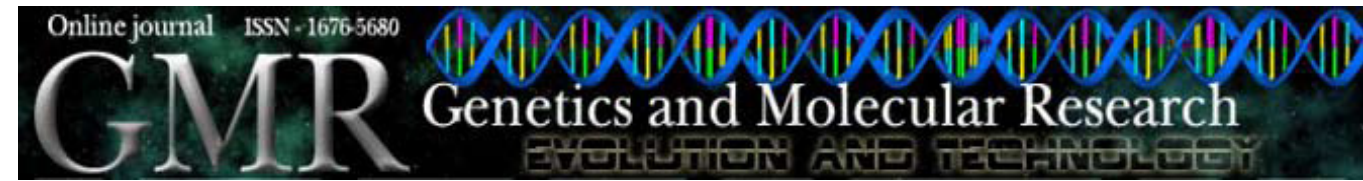

\title{
Phylogenetic relationships among Saccharum clones in Pakistan revealed by RAPD markers
}

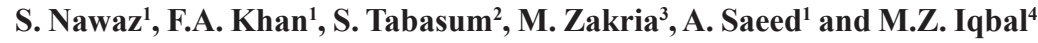 \\ ${ }^{1}$ Department of Plant Breeding and Genetics, University of Agriculture, \\ Faisalabad, Pakistan \\ ${ }^{2}$ University College of Agriculture, Sargodha, Pakistan \\ ${ }^{3}$ Department of Mathematics and Statistics, University of Agriculture, \\ Faisalabad, Pakistan \\ ${ }^{4}$ Ayub Agriculture Research Institute, Faisalabad, Pakistan \\ Corresponding author: A. Saeed \\ E-mail: drasifpbg@gmail.com
}

Genet. Mol. Res. 9 (3): 1673-1682 (2010)

Received May 5, 2010

Accepted June 14, 2010

Published August 24, 2010

DOI 10.4238/vol9-3gmr903

\begin{abstract}
Forty sugarcane genotypes (clones), including elite lines, commercial cultivars of Saccharum officinarum and S. barberi clones, were fingerprinted with 30 RAPD markers, using a PCR-based marker assay. The genetic distance for RAPD data was determined according to Nei, and relationships between accessions were graphed in a dendrogram. Genetic distance values ranging from 16.2 to $86.3 \%$ were observed among the 40 sugarcane accessions. The lowest genetic distance was found between genotypes US-406 and US-186. These two genotypes differed from each other in only 25 bands with 15 different primers. Genotypes Col-54 and CP-72-2086 were the second most similar group, with a genetic distance of $19.46 \%$. The most dissimilar of all the accessions were CP-77-400 and US-133, with a genetic distance of $86.3 \%$. RAPD fingerprints help sugarcane breeders clarify the genetic pedigree of commercial sugarcane varieties and can be used to evaluate the efficiency of conventional breeding methods.
\end{abstract}

Key words: DNA marker; RAPD; Genetic distance; Sugarcane; Fingerprinting 


\section{INTRODUCTION}

Sugarcane is a large perennial grass belonging to the genus Saccharum, which is an important component of the grass family Poaceae and the tribe Andropogoneae. Six Saccharum species, namely Saccharum officinarum, S. robustum, S. spontaneum, S. barberi, S. sinense, and S. edule, along with Erianthus, Miscanthus, Narenga, and Sclerostachya are so closely related that Mukherjee (1957) referred to them as the "Saccharum complex".

The evolution of cultivated sugarcane could have resulted through several stages of intergeneric and interspecific hybridization involving the above core taxa, followed by polyploidy and natural- and human-mediated selection. In sugarcane, the assessment of genetic diversity existing among the species and related genera is particularly important, since a major breakthrough in sugarcane varietal improvement was obtained through distant hybridization. Although varietal diversification is apparent among the current commercial varieties, an assessment of their genetic diversity is lacking. The extent of genetic diversity among the genetic materials has been estimated by adopting various methods over a period of time using a wider range of simply and complexly inherited traits. Although morphological and isozyme markers have been employed in assessing the underlying genetic diversity of a species, the accuracy of such assessment is questionable. Considering the problems associated with morphological and isozyme markers, research on genetic diversity analysis has searched for alternative tools. Advances in molecular biology techniques have provided the basis for uncovering virtually unlimited numbers of DNA markers. During the last decade, several molecular marker systems such as restriction fragment length polymorphism (RFLP), microsatellite, random amplified polymorphic DNA (RAPD), and amplified fragment length polymorphism (AFLP) were developed and used for the estimation of genetic diversity (Wang et al., 1992; Stiles et al., 1993; Orozco-Castillo et al., 1994; Nakajima et al., 1998; Hokanson et al., 1998; Aggarwal et al., 1999; Angiolillo et al., 1999; Barker et al., 1999).

Polymerase chain reaction (PCR)-based methods such as RAPD are increasingly being used in the analysis of genetic diversity in crop plants, because of the relative ease with which PCR assays can be carried out compared to RFLPs. Both genomic and organellar RFLP along with PCRbased markers have been used to study the variability and diversity among Saccharum species and hybrid populations (D’Hont et al., 1993; Al-Janabi et al., 1994; Lu et al., 1994; Sobral et al., 1994; Burnquist et al., 1995; Harvey and Botha, 1996; Nair et al., 1999). These studies have been useful in assessing the genetic diversity among Saccharum species and hybrids.

This paper reports the results of a study of the genetic diversity among 40 genotypes including currently cultivated sugarcane varieties, elite lines and the wild $S$. barberi species, as revealed by RAPD molecular markers. RAPD fingerprints help sugarcane breeders in the identification of mislabeled varieties during field trials (Lande and Thompson, 1990), markerassisted selection of true cross progeny at seedling stage, and evaluation of the extent of selfpollination in conventional crossing procedure (Stuber et al., 1999).

\section{MATERIAL AND METHODS}

\section{Plant genotypes and DNA extraction}

Forty sugarcane genotypes (clones), including elite lines, commercial cultivars of $S$. officinarum and clones of $S$. barberi were selected for the study of genetic diversity (Table 1). 
The parameters yield potential, maturity trend, ratoonability, salt tolerance, and disease resistance were used as selection criteria for the above mentioned genotypes (data not shown). DNA was extracted from shoot apical meristems, which were ground into fine powder in liquid nitrogen. Genomic DNA was extracted by the CTAB method (Hoisington et al., 1994) with minor modifications for sugarcane.

\begin{tabular}{|c|c|c|c|c|}
\hline Sr. No. & Genera and species & Cultivar & Origin & Source of collection \\
\hline 1 & Saccharum barberi & No. $61 / 77$ & Unknown & UAF \\
\hline 2 & & AUS10/72 & Australia & UAF \\
\hline 3 & & No. $21 / 77$ & Unknown & UAF \\
\hline 4 & & Katha & India & UAF \\
\hline 5 & Saccharum officinarum & No. 46 & Unknown & UAF \\
\hline 6 & & No. 64 & Unknown & UAF \\
\hline 7 & & СР-77-400 & Canal point & UAF \\
\hline 8 & & СР-43-33 & Canal point & UAF \\
\hline 9 & & CPF-232 & Canal point & UAF \\
\hline 10 & & L-118 & Louisiana & UAF \\
\hline 11 & & HSF-242 & Unknown & UAF \\
\hline 12 & & SPF-234 & Brazil & UAF \\
\hline 13 & & No. 61 & Unknown & UAF \\
\hline 14 & & Col-54 & Colombia & UAF \\
\hline 15 & & CP-72-2086 & Canal point & UAF \\
\hline 16 & & HSF-240 & Unknown & UAF \\
\hline 17 & & Coj-64 & Early Indian & UAF \\
\hline 18 & & S-97-US-297 & Unknown & UAF \\
\hline 19 & & $31 / 77$ & Unknown & UAF \\
\hline 20 & & AUS-11/72 & Australia & UAF \\
\hline 21 & & SASG-26 & Pakistan & UAF \\
\hline 22 & & CPF-235 & Canal point & UAF \\
\hline 23 & & SPF-213 & Unknown & UAF \\
\hline 24 & & Col-72 & Colombia & UAF \\
\hline 25 & & Coj-84 & India & UAF \\
\hline 26 & & BF-129 & Unknown & UAF \\
\hline 27 & & CPF-237 & Canal point & UAF \\
\hline 28 & & Triton & India & UAF \\
\hline 29 & & TCP-81 & Brazil & UAF \\
\hline 30 & & No. $41 / 77$ & Unknown & UAF \\
\hline 31 & & US-50 & USA & AARI \\
\hline 32 & & US-173 & USA & AARI \\
\hline 33 & & US-133 & USA & AARI \\
\hline 34 & & US-113 & USA & AARI \\
\hline 35 & & US-64 & USA & AARI \\
\hline 36 & & US-234 & USA & AARI \\
\hline 37 & & US-406 & USA & AARI \\
\hline 38 & & US-186 & USA & AARI \\
\hline 39 & & US- 123 & USA & AARI \\
\hline 40 & & US-109 & USA & AARI \\
\hline
\end{tabular}

$\mathrm{UAF}=$ University of Agriculture, Faisalabad; AARI = Ayub Agriculture Research Institute.

\section{PCR amplification}

PCR conditions were optimized in a Gene Amp 2700 thermal cycler (Applied Biosystems, Foster City, CA, USA). PCR for RAPD analysis was performed in a $25-\mu \mathrm{L}$ reaction volume containing $8.3 \mu \mathrm{L} \mathrm{d}_{3} \mathrm{H}_{2} \mathrm{O}, 2.5 \mu \mathrm{L} \mathrm{10X} \mathrm{PCR} \mathrm{buffer,} 2.5 \mu \mathrm{L}$ gelatin, $3 \mu \mathrm{L} \mathrm{MgCl}_{2}, 4$ $\mu \mathrm{L}$ of each dNTP (Fermentas Inc., MD, USA), $0.2 \mu \mathrm{L}$ Taq DNA polymerase (Fermentas), 
$2.5 \mu \mathrm{L}$ template DNA and $2 \mu \mathrm{L}$ of each primer. The reaction mixes were subjected to the following protocol: initial denaturation at $94^{\circ} \mathrm{C}$ for $5 \mathrm{~min}$, followed by 35 cycles, each consisting of 1 min denaturation at $94^{\circ} \mathrm{C}, 2$ min annealing at $36^{\circ} \mathrm{C}$ and 1 min extension at $72^{\circ} \mathrm{C}$, with a final extension at $72^{\circ} \mathrm{C}$ for $5 \mathrm{~min}$. Amplification products were mixed with $3 \mu \mathrm{L} 6 \mathrm{X}$ loading dye $(0.25 \%$ bromophenol blue, $0.25 \%$ xylene cyanole, and $40 \%$ sucrose $)$ spun for few minutes in a centrifuge before loading. The PCR products $(7 \mu \mathrm{L})$ were loaded in each well of a $1.2 \%$ agarose gel made with $0.5 \mathrm{X}$ TBE buffer and $0.5 \mu \mathrm{g} / \mathrm{mL}$ ethidium bromide, and electrophoresed at $90 \mathrm{~W}$ for $2 \mathrm{~h}$. The gels were dried and photographed under UV. All bands that were well resolved and unambiguous were scored for presence (1) or absence (0) in the 40 genotypes.

\section{Data analysis}

The data on bands generated by the 30 primers were selected for the analysis of genetic diversity. The bands were counted by starting from the top of the lanes to their bottom. The data of the primers were used to estimate dissimilarity based on the number of unshared amplified products, and a dissimilarity matrix was generated using Nei's similarity indices (Nei, 1978). In addition, population relationships were inferred using the unweighted pair group method with arithmetic mean (UPGMA) clustering method using the Popgen software (version 3.5).

\section{RESULTS}

\section{RAPD polymorphism}

Ninety-two and five percent polymorphic bands were estimated since 219 of 238 fragments were polymorphic with 30 primers used among the 40 sugarcane accessions. The rest of the 19 bands were monomorphic in the 40 accessions. In the present study, the 40 sugarcane accessions appeared to show a difference/variability with the 30 primers used. Although none of the primers was individually so informative as to differentiate all accessions, highly polymorphic profiles were obtained with primers such as GL Decamer A-5, GL Decamer A-9, GL Decamer B-3, GL Decamer B-11, GL Decamer B-I7, and GL Decamer D-1. Therefore, it may be concluded from the present results that RAPD markers can be used for the identification of sugarcane accessions.

\section{Genetics distance between the accessions}

The genetic distance for RAPD data using 40 sugarcane accessions was constructed according to Nei (1978), and relationships between accessions were portrayed graphically in the form of a dendrogram in Figure 1. Genetic distance values ranging from 16.21 to $86.33 \%$ were observed among the 40 sugarcane accessions. The lowest genetic distance was 16.21 as seen in genotypes US-406 and US-186. These two genotypes differed from each other only in 25 bands with 15 different primers. The genotypes Col-54 and CP-72-2086 were the second similar group with a genetic distance of $19.46 \%$. The most dissimilar of all the accessions was CP-77-400 and US-133 with a genetic distance of $86.33 \%$. 


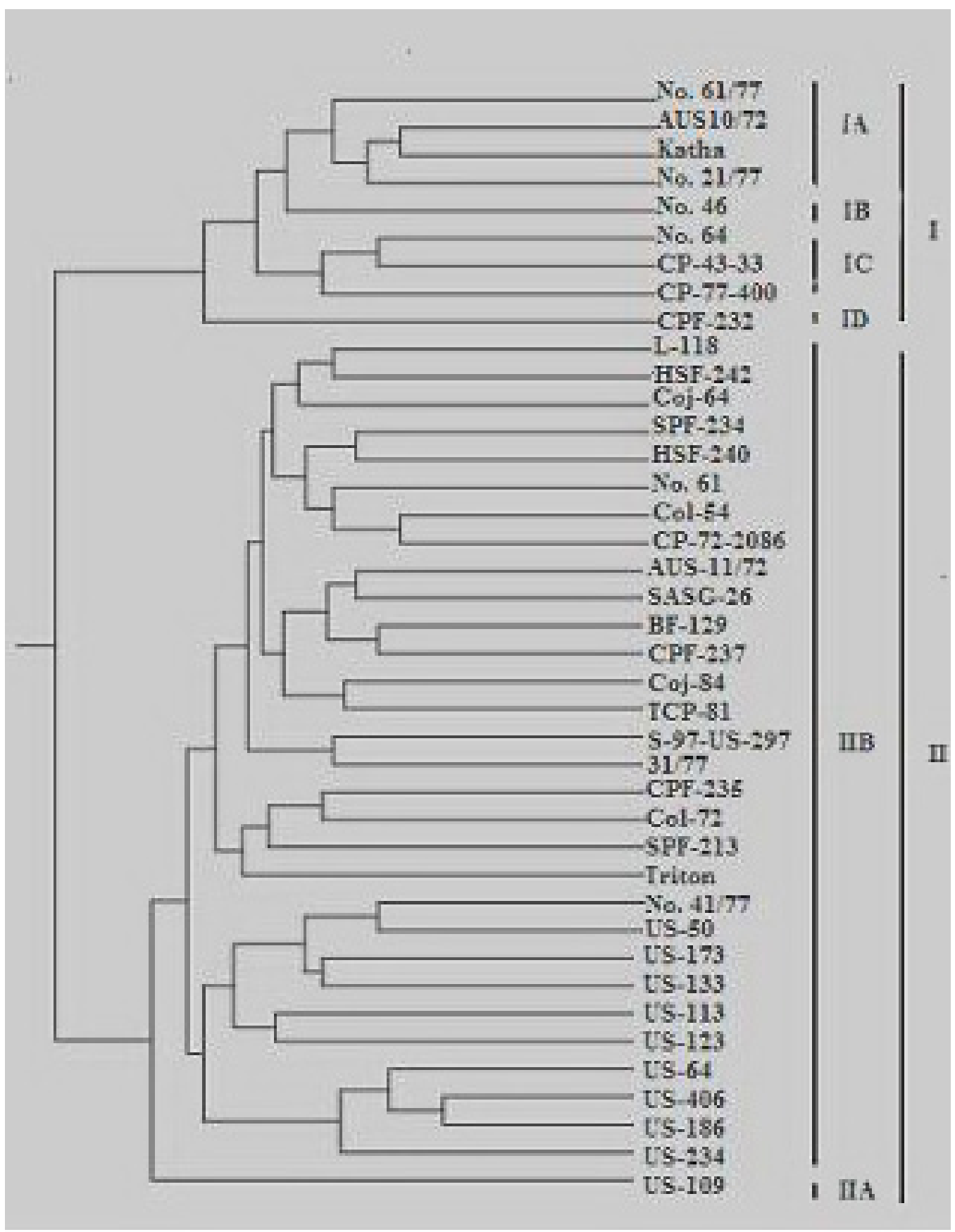

Figure 1. Dendrogram of 40 sugarcane accessions constructed from RAPD data using unweighted pair group method with arithmetic mean (UPGMA) based on Nei's (1978) genetic distance. 


\section{Clustering pattern}

The cluster analysis based on dissimilarity values classified all sugarcane accessions into two major groups (I and II) (Figure 1). The first major group was further divided into four clusters IA, IB, IC, and ID. Cluster IA consisted of four $S$. barberi accessions, namely No. 61/77, AUS10/72, No. 21/77, and Katha, while Cluster IB consisted of only one S. officinarum accession, namely No. 46. Cluster IC consisted of three accessions, namely No. 64, CP-77-400 and CP-43-33. Cluster ID consisted of only one accession, namely CPF-232. The second major group was further divided into Groups IIA and IIB. Group IIA consisted of only one genotype, namely US-109. Group IIB was again divided into seven sub-groups, $\mathrm{IIB}_{1}, \mathrm{IIB}_{2}, \mathrm{IIB}_{3}, \mathrm{IIB}_{4}, \mathrm{IIB}_{5}, \mathrm{IIB}_{6}$, and IIB I Sub-group IIB $_{1}$ consisted of four elite lines US-64, US-234, US-406, and US-186, while sub-group IIB ${ }_{2}$ was further divided into two sub-clusters. Group $\mathrm{IIB}_{2}$ a comprised two elite lines, US-113 and US-123. Group $\mathrm{IIB}_{2} \mathrm{~b}$ comprised four elite lines No. 41/77, US-50, US-173, and US-133. Sub-group IIB $_{3}$ consisted of four sugarcane accessions, namely CPF-235, SPF-213, Col-72, and Triton. Sub-group $\mathrm{IIB}_{4}$ consisted of two sugarcane accessions, namely S-97-US-297 and No. 31/77. Sub-group IIB $_{5}$ was further divided into two sub-clusters. Group $\mathrm{IIB}_{5}$ a comprised two accessions, namely Coj-84 and TCP-81. Group IIB $_{5}$ b comprised four accessions, namely AUS-11/72, SASG26, BF-129, and CPF-237. Sub-group IIB ${ }_{6}$ was further divided into two sub-clusters. Group $\mathrm{IIB}_{6}$ a comprised three accessions, namely No. 61, Col-54 and CP-72-2086. Group IIB 6 b comprised two accessions, namely SPF-234 and HSF-240. Sub-group IIB $_{7}$ consisted of three sugarcane accessions, namely L-118, HSF-242 and Coj-64.

\section{DISCUSSION}

For achieving improved productivity in the sugarcane crop, it is essential to maintain a high degree of genetic diversity among the commercial varieties and breeding populations. Although diversification is apparent among the current germplasm collection, an assessment of its genetic diversity is lacking. The present investigation reports the results of a study on the genetic diversity among 40 accessions of sugarcane belonging to $S$. officinarum L. and $S$. barberi as revealed by RAPD. By using RAPD as genetic markers, as high as $92.05 \%$ polymorphic bands were detected in 40 accessions of sugarcane. Nair et al. (2002) investigated genetic diversity in prominent Indian sugarcane varieties with $63.74 \%$ polymorphism. Burner et al. (1997) compared the genetic diversity of North American and an Old World member of Saccharum with $31.44 \%$ polymorphism across the taxa. Polymorphism revealed by RAPD could be a result of nucleotide changes at the primer annealing site or due to addition or deletion between two priming sites, which results in different lengths of the amplification products (Williams et al., 1990).

Genetic diversity is commonly measured by genetic distance or genetic similarity, both of which imply that there are either differences or similarities at the genetic level (Weir, 1990). The genetic distance of 40 accessions ranging from 0.16 to 0.86 (Table 2) with an average of 0.51 suggests that the level of genetic diversity among the sugarcane accessions is moderate. In several other studies, elite sugarcane (Saccharum hybrids) germplasm also showed genetic diversity (Arceneaux, 1967; Harvey et al., 1994; Harvey and Botha, 1996). Harvey and Botha (1996) reported similarities as high as 77-95\% among 20 elite varieties. 
Phylogenetic relationships among Saccharum species

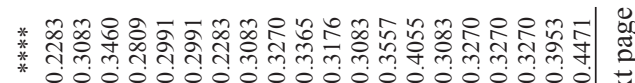

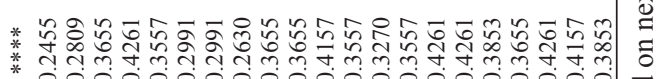

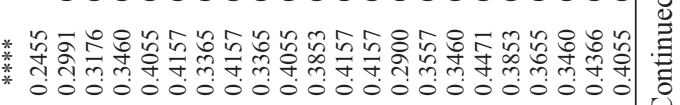

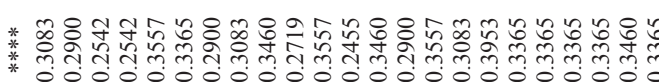

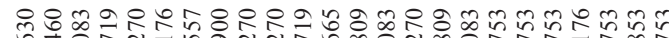

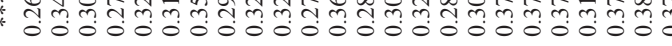

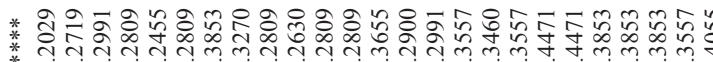

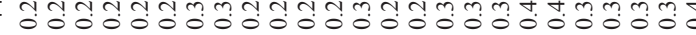

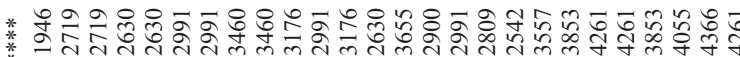

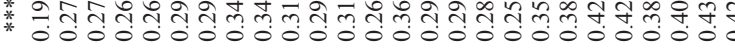

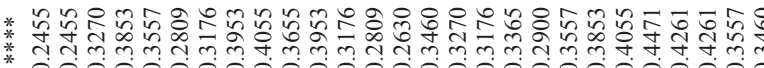

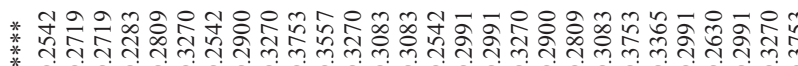

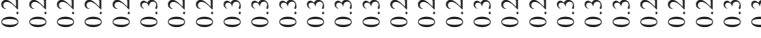

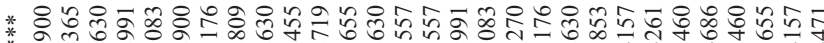

14. : * :

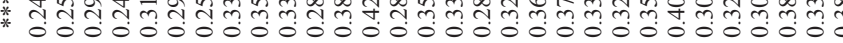
* \&

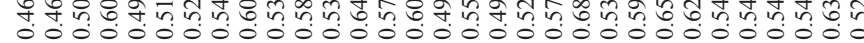

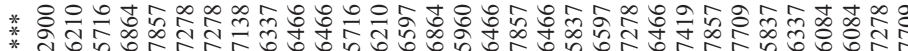

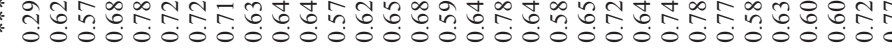
:ำด : A.

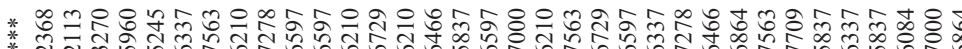

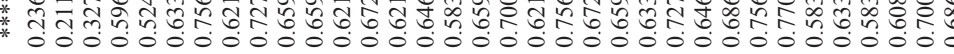

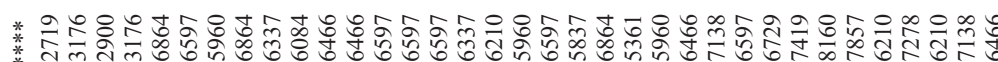

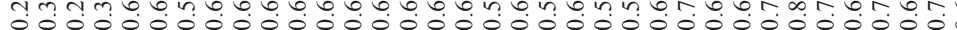

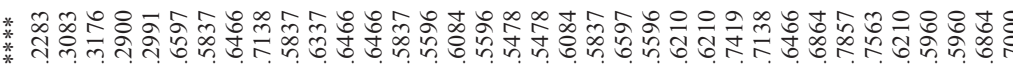
तु * 유슈

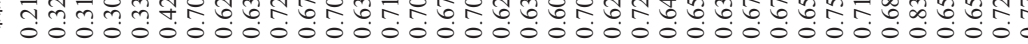

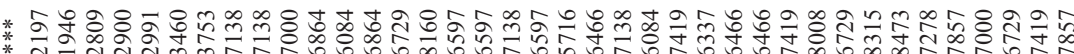

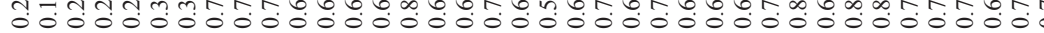

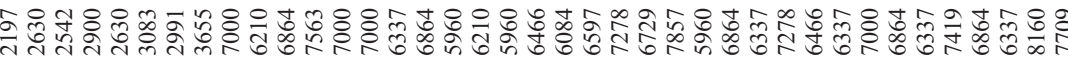

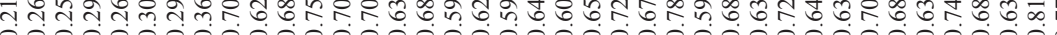

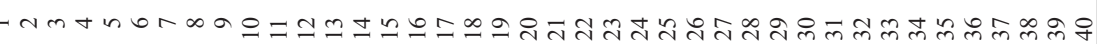


S. Nawaz et al.

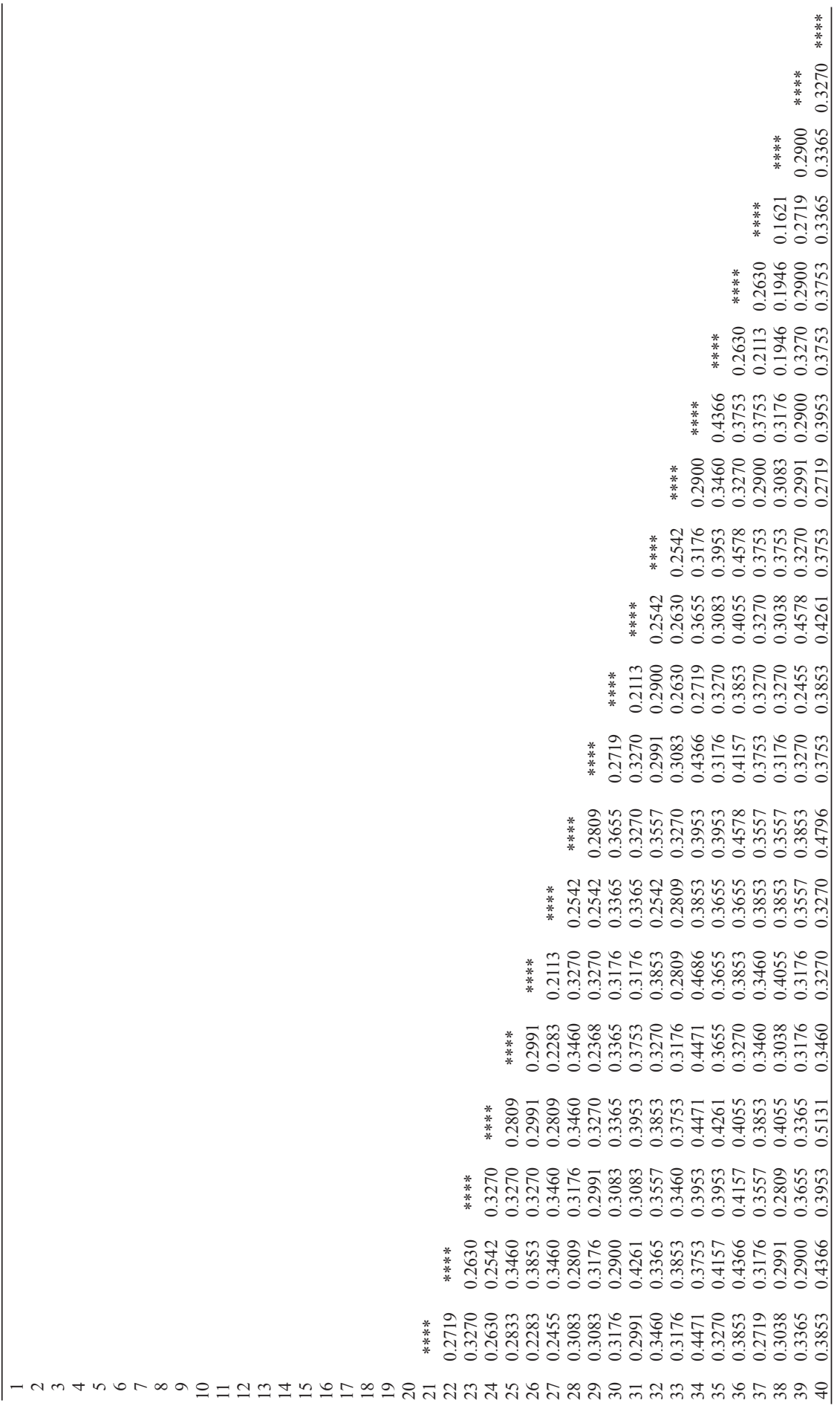


In the present study, the four $S$. barberi clones used formed clusters with $S$. officinarum clones, instead of forming distinct/separate clusters, supporting the assumption of Pan et al. (2003) that sugarcane cultivars (Saccharum hybrids) are aneupolyploid hybrids of $S$. officinarum, S. barberi, S. sinense, and S. robustum. Nair et al. (1999) hypothesized that only two species, $S$. robustum and $S$. spontaneum, are the progenitors of modern sugarcane, that S. officinarum may be derived from $S$. robustum, and that $S$. barberi and $S$. sinense are cultivated forms of interspecific hybrids between $S$. spontaneum and $S$. officinarum. The above hypothesis is supported by the present study as $S$. barberi clones (No. 61/77, AUS10/72, No. 21/77, and Katha) and S. officinarum clones formed a different cluster in Group I. However, the genetic distance between $S$. barberi clone No. 61/77 and another clone in that group was $0.21,0.25,0.26,0.29,0.30$, and 0.36 , respectively.

Thus, the $S$. officinarum varieties and $S$. barberi clones have genetic distances in the range of 0.21 to 0.36 , where the level of genetic diversity between these sugarcane accessions is somewhat moderate. Other accessions are grouped under different clusters and US-109 is the most distinct accession, forming a separate cluster. The mean genetic distance among the 40 accessions in this study was $51 \%$, implying that the genetic diversity among the genotypes is limited. This is probably due to the lack of parental diversity, where a few clones are themselves related, contributing to the parentage of these varieties. Thus, conscious efforts are to be made to diversify the parental genetic base to ensure high genetic variability among the cultivated varieties and elite lines. New sources from the interspecific/intergeneric hybrid gene pool need to be used along with proven parents to generate the variability that will be both commercially viable and genetically diverse. Elite lines can also be further improved by arranging their cross with $S$. barberi, as this wild species contains high tillering ability and disease resistance.

\section{REFERENCES}

Aggarwal RK, Brar DS, Nandi S, Huang N, et al. (1999). Phylogenetic relationships among Oryza species revealed by AFLP markers. Theor. Appl. Genet. 98: 1320-1328.

Al-Janabi SM, McClelland M, Petersen C and Sobral BWS (1944). Phylogenetic analysis of organellar DNA sequences in the Andropogoneae: Saccharinae. Theor. Appl. Genet. 88: 933-944.

Angiolillo A, Mencuccini M and Baldoni L (1999). Olive genetic diversity assessed using amplified fragment length polymorphisms. Theor. Appl. Genet. 98: 411-421.

Arceneaux G (1967). Cultivated sugarcane of the world and their botanical derivation. Proc. Int. Soc. Sugar Cane Technol. 12: $844-845$

Barker JH, Matthes M, Arnold GM, Edwards KJ, et al. (1999). Characterisation of genetic diversity in potential biomass willows (Salix spp.) by RAPD and AFLP analyses. Genome 42: 173-183.

Burner DM, Pan YB and Webster RD (1997). Genetic diversity of North American and Old World Saccharum assessed by RAPD analysis. Genet. Resour. Crop Evol. 44: 235-240.

Burnquist WL, Sorrelles ME and Tanksley S (1995). Characterization of genetic variability in Saccharum germplasm by means of restriction fragment length polymorphism (RFLP) analysis. Proc. Int. Soc. Sugar Cane Technol. 21: 355-365.

D’Hont A, Lu YH, Feldmann P and Glaszmann JC (1993). Cytoplasmic diversity in sugar cane revealed by heterologous probes. Sugar Cane 1: 12-15.

Harvey H, Huckett BI and Botha FC (1994). Use of polymerase chain reaction and random amplification of polymorphic DNAs for the determination of genetic distances between 21 sugarcane varieties. Proc. S. Afr. Sugar Technol. Assoc. 68: $36-40$.

Harvey M and Botha FC (1996). Use of PCR-based methodologies for the determination of DNA diversity between Saccharum varieties. Euphytica 89: 257-265.

Hoisington D, Khairallah M and González D (1994). Laboratory Protocols: CIMMYT Applied Molecular Genetics Laboratory. CIMMYT, Mexico.

Hokanson SC, Szewc-McFadden AK, Lamboy WF and McFerson JR (1998). Microsatellite (SSR) markers reveal genetic 
identities, genetic diversity and relationships in a Malus x domestica borkh. core subset collection. Theor. Appl. Genet. 97: 671-683.

Lande R and Thompson R (1990). Efficiency of marker-assisted selection in the improvement of quantitative traits. Genetics 124: 743-756.

Lu YH, D’Hont A, Walker DIT, Rao PS, et al. (1994). Relationships among ancestral species of sugarcane revealed with RFLP using single-copy maize nuclear probes. Euphytica 78: 7-18.

Mukherjee SK (1957). Origin and distribution of Saccharum. Bot. Gaz. 119: 55-61.

Nair NV, Nair S, Sreenivasan TV and Mohan M (1999). Analysis of genetic diversity and phylogeny in Saccharum and related genera using RAPD markers. Genet. Resour. Crop Evol. 46: 73-79.

Nair NV, Selvi A, Sreenivasan TV and Pushpalatha KN (2002). Molecular diversity in Indian sugarcane cultivars as revealed by randomly amplified DNA polymorphisms. Euphytica 127: 219-225.

Nakajima Y, Oeda K and Yamamoto T (1998). Characterization of genetic diversity of nuclear and mitochondrial genomes in Daucus varieties by RAPD and AFLP. Plant Cell Rep. 17: 848-853.

Nei M (1978). Estimation of average heterozygosity and genetic distance from a small number of individuals. Genetics 89: $583-590$

Orozco-Castillo C, Chalmers KJ, Waugh R and Powell W (1994). Detection of genetic diversity and selective gene introgression in coffee using RAPD markers. Theor. Appl. Genet. 87: 934-940.

Pan Y, Miller JD, Schnell Ii RJ, Richard EP Jr, et al. (2003). Application of Microsatellite and RAPD Fingerprints in the Florida Sugarcane Variety Program. In: Plant and Animal Genome Conference, Town \& Country Convention Center, January 13-17, San Diego, 43.

Sobral BWS, Braga DPV, LaHood ES and Keim P (1994). Phylogenetic analysis of chloroplast restriction enzyme site mutations in the Saccharinae Griseb. subtribe of the Andropogoneae Dumort. tribe. Theor. Appl. Genet. 87: 843-853.

Stiles JI, Lemme C, Sondur S, Morshid MB, et al. (1993). Using randomly amplified polymorphic DNA for evaluating genetic relationships among papaya cultivars. Theor. Appl. Genet. 85: 697-701.

Stuber CW, Polacco M and Senior ML (1999). Synergy of empirical breeding, marker-assisted selection, and genomics to increase crop yield potential. Crop. Sci. 39: 1571-1583.

Wang ZY, Second G and Tanksley SD (1992). Polymorphism and phylogenetic relationships among species in the genus Oryza as determined by analysis of nuclear RFLPs. Theor. Appl. Genet. 83: 565-581.

Weir BS (1990). Genetic Data Analysis: Methods for Discrete Population Genetic Data. Sinauer Associates Inc., Sunderland.

Williams JG, Kubelik AR, Livak KJ, Rafalski JA, et al. (1990). DNA polymorphisms amplified by arbitrary primers are useful as genetic markers. Nucleic Acids Res. 18: 6531-6535. 